\title{
Minimalistic control of biped walking in rough terrain
}

\section{Journal Article}

Author(s):

lida, Fumiya; Tedrake, Russ

Publication date:

2010

Permanent link:

https://doi.org/10.3929/ethz-b-000016976

Rights / license:

In Copyright - Non-Commercial Use Permitted

Originally published in:

Autonomous Robots 28(3), https://doi.org/10.1007/s10514-009-9174-3 


\title{
Minimalistic control of biped walking in rough terrain
}

\author{
Fumiya Iida $\cdot$ Russ Tedrake
}

Received: 7 April 2009 / Accepted: 17 December 2009 / Published online: 7 January 2010

(C) Springer Science+Business Media, LLC 2010

\begin{abstract}
Toward our comprehensive understanding of legged locomotion in animals and machines, the compass gait model has been intensively studied for a systematic investigation of complex biped locomotion dynamics. While most of the previous studies focused only on the locomotion on flat surfaces, in this article, we tackle with the problem of bipedal locomotion in rough terrains by using a minimalistic control architecture for the compass gait walking model. This controller utilizes an open-loop sinusoidal oscillation of hip motor, which induces basic walking stability without sensory feedback. A set of simulation analyses show that the underlying mechanism lies in the "phase locking" mechanism that compensates phase delays between mechanical dynamics and the open-loop motor oscillation resulting in a relatively large basin of attraction in dynamic bipedal walking. By exploiting this mechanism, we also explain how the basin of attraction can be controlled by manipulating the parameters of oscillator not only on a flat terrain but also in various inclined slopes. Based on the simulation analysis, the proposed controller is implemented in a real-world robotic platform to confirm the plausibility of the approach. In addition, by using these basic principles of self-stability and
\end{abstract}

\footnotetext{
F. Iida $(\bowtie)$

Institute of Robotics and Intelligent Systems, Swiss Federal Institute of Technology Zurich, LEO D 9.2, Leonhardstrasse 27, 8092 Zurich, Switzerland

e-mail: fumiya.iida@mavt.ethz.ch

F. Iida

e-mail: iida@csail.mit.edu

R. Tedrake

Computer Science and Artificial Intelligence Laboratory,

Massachusetts Institute of Technology, 32 Vassar Street,

Cambridge, MA 02139, USA

e-mail: russt@mit.edu
}

gait variability, we demonstrate how the proposed controller can be extended with a simple sensory feedback such that the robot is able to control gait patterns autonomously for traversing a rough terrain.

Keywords Dynamic legged locomotion - Biped robot . Passive dynamics · Compass gait model

\section{Introduction}

Since the pioneering work of the Passive Dynamic Walkers (PDWs: McGeer 1990; Collins et al. 2001), the problem of dynamic walking has attracted a number of researchers in order to understand the nature of legged locomotion in biological systems and to improve locomotion capabilities of legged robots. If compared with fully actuated legged robots, the use of passive dynamics is expected not only to substantially increase energy efficiency but also to obtain additional insights into the design principes of legged locomotion in nature. Previously it has been demonstrated that the use of passive dynamics leads to energetically efficient dynamic locomotion (Collins et al. 2005; Wisse and van Frankenhuyzen 2003; Tedrake et al. 2004; Tedrake 2004) as well as mechanically self-stabilizing locomotion dynamics (Hobbelen and Wisse 2008; Iida et al. 2008). Despite these demonstrations, control of the PDWs appears to be a challenging problem because of the nonlinearity originated in complex mechanical dynamics, and the locomotion capabilities of these robots are still restricted on a level ground.

In order to obtain an in-depth understanding of dynamic bipedal walking, the so-called compass gait walking model (also known as the simplest walking model) has been intensively studied (Kajita and Espiau 2008). An important aspect 
of this model lies in the fact that it is irreducibly simple and analytically tractable, which enable us systematically investigate both mechanical interactions and dynamic behavior control. Previously, the compass gait model was investigated in terms of mechanical interactions in a passive regime (McGeer 1990; Garcia et al. 1998; Goswami et al. 1998; Su and Dingwell 2007), and its variations were developed for investigating, for example, knee dynamics and locomotion stability (van der Linde 1999; Miyakoshi and Cheng 2004; Asano et al. 2007; Harata et al. 2007; Kinugasa et al. 2008), shapes and actuation of foot segments (Kuo 2002; Ono et al. 2004; Adamczyk et al. 2006; Kim et al. 2007; Kwan and Hubbard 2007), mass distribution (Hass et al. 2006), and lateral balancing (Kuo 1999). Control architectures for the compass gait model have also been studied with respect to energy based optimal control (McGeer 1988; Goswami et al. 1997; Asano et al. 2000; Spong 2003; Spong and Bhatia 2003; Asano et al. 2004; Pekarek et al. 2007), phase resetting mechanisms and nonlinear oscillators (Kurz and Stergiou 2005; Aoi and Tsuchiya 2005, 2006, 2007), and control optimization in rough terrains (Pratt et al. 2001; Byl and Tedrake 2008a, 2008b). Through these previous studies on the compass gait model and its variations, we have gained accumulated knowledge about the stability and controllability, whereas most of the studies above were conducted in flat environment or only in simulation.

From this perspective, the primary goal of this article is to investigate a minimalistic control architecture for the compass gait model that can be used for locomotion in rough terrains. More specifically, the main contribution of this article lies in the following two intrinsic characteristics of the proposed control scheme, which have not been reported in the past. First, we show that the compass gait model has an intrinsic self-stability in the locomotion of various inclined slopes, if a specific open-loop oscillation is applied to the hip motor. We identified that the self-stability is originated in the "phase locking" mechanism, that is, a mechanism which compensates undesired phase delays between walking dynamics and motor oscillation without any explicit control. This mechanism is particularly beneficial if compared with the previously proposed control architectures, because no state feedback is necessary (including no need of detecting stance/swing phases). And second, this article also shows how the phase locking mechanism can be exploited further, and facilitate the design of higher-level controller for the locomotion planning in rough terrains. The proposed minimalistic control approach has an additional intrinsic characteristics, that is, walking dynamics of the compass gait model can be harnessed around specific nominal trajectories, which are uniquely determined by the parameters of the open-loop oscillator. Namely, different sets of parameters in the oscillator (e.g. oscillator frequency and amplitude) result in different walking trajectories (e.g. different stride length), and such a characteristics can be eventually used for the control of footholds in rough terrains.

In this article, the main results are presented through both simulation analyses and real-world experiments. In simulation experiments, we intend to generalize the aforementioned arguments for the typical theoretical model of compass gait, and the real-world experiments should convince the applicability of the arguments in the real-world robots. In the next section, we first explain the simulation model that is used to analyze the details of phase locking mechanism. In Sect. 3, the experimental platform and method are explained. Here we analyze walking dynamics of the robotic platform that we developed, and compare them with those of the theoretical model (Iida and Tedrake 2009). Section 4 shows the application of the proposed control approach. Namely we extend the minimalistic control architecture with a minimum sensory feedback, and demonstrate that the proposed controller can take advantage of the intrinsic stability and gait variability to autonomously navigate through a relatively complex rough terrain. Finally in Sect. 5, we summarize the contributions and implications of the main results presented in this article.

\section{Control of a compass gait model}

For a systematic investigation of the minimalistic control architecture, this section first introduces the compass gait model and basic assumptions of the controller. Then the underlying mechanism of self-stability is explained through a set of simulation experiments.

\subsection{Compass gait model}

The compass gait model (Fig. 1) consists of two sets of dynamics, i.e. a continuous dynamics of swing leg and a transition dynamics that occurs at the event of touchdown and switching of the swing and stance legs.

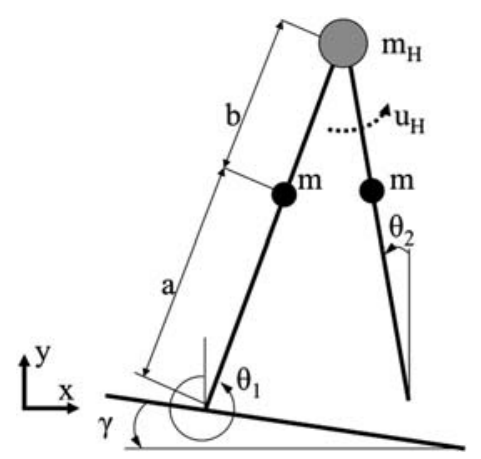

Fig. 1 Compass gait model. A point mass $m_{H}$ is defined at the hip joint, which is actuated by motor torque $u_{H}$. Black circles denote the centers of leg mass, which are determined by $a$ and $b$ 
Table 1 Specification of simulation model

\begin{tabular}{lll}
\hline Symbol & Description & Value \\
\hline$a$ & Lower leg segment & $0.5 \mathrm{~m}$ \\
$b$ & Upper leg segment & $0.5 \mathrm{~m}$ \\
$m$ & Mass of leg & $5.0 \mathrm{~kg}$ \\
$m_{H}$ & Mass of body & $5.0 \mathrm{~kg}$ \\
$g$ & Gravitational constant & $9.8 \mathrm{~m} / \mathrm{s}^{2}$ \\
\hline
\end{tabular}

The swing-leg dynamics of the compass gait model can be described as follows.

$$
\mathbf{M}(\mathbf{q}) \ddot{\mathbf{q}}+\mathbf{C}(\mathbf{q}, \dot{\mathbf{q}}) \dot{\mathbf{q}}+\mathbf{G}(\mathbf{q})=\mathbf{B u},
$$

$$
\begin{aligned}
\mathbf{M}(\mathbf{q}) & =\left[\begin{array}{cc}
m b^{2} & -m b l \cos \left(\theta_{1}-\theta_{2}\right) \\
-m b l \cos \left(\theta_{1}-\theta_{2}\right) & m a^{2}+m_{H} l^{2}+m l^{2}
\end{array}\right] \\
\mathbf{C}(\mathbf{q}, \dot{\mathbf{q}}) & =\left[\begin{array}{cc}
0 & m b l \sin \left(\theta_{1}-\theta_{2}\right) \dot{\theta_{1}} \\
-m b l \sin \left(\theta_{1}-\theta_{2}\right) \dot{\theta}_{2} & 0
\end{array}\right], \\
\mathbf{G}(\mathbf{q}) & =\left[\begin{array}{cc}
-m g a \sin \theta_{1}-m_{H} g l \sin \theta_{1}-m g l \sin \theta_{1}
\end{array}\right], \\
-\mathbf{B} & =\left[\begin{array}{cc}
1 & 1 \\
0 & -1
\end{array}\right]
\end{aligned}
$$

where $\mathbf{q}=\left[\theta_{1}, \theta_{2}\right]^{T}, \mathbf{u}=\left[u_{H}, 0\right]^{T}\left(u_{H}\right.$ is torque generated by the hip actuator), and $l=a+b$ (see Table 1 for specifications).

When the state variables satisfy $\theta_{1}-\theta_{2}=\gamma$, the swingleg dynamics is terminated, and the collision dynamics is computed as follows. At the ground contact of the swing leg and switching to the stance leg, the compass gait model assumes the conservation of angular momentum around the hip joint and the toe of the swing leg.

$$
\begin{aligned}
& \mathbf{Q}_{p} \dot{\mathbf{q}}^{+}=\mathbf{Q}_{m} \dot{\mathbf{q}}^{-} \\
& \mathbf{Q}_{p}=\left[\begin{array}{c}
m b^{2}-m b l \cos 2 \alpha \\
m b^{2}
\end{array},\right. \\
& \left.\begin{array}{c}
m l^{2}+m_{H} l^{2}+m a^{2}-m b l \cos 2 \alpha \\
-m b l \cos 2 \alpha
\end{array}\right], \\
& \mathbf{Q}_{m}=\left[\begin{array}{cc}
-m a b & -m a b+\left(m_{H} l^{2}+2 m a l\right) \cos 2 \alpha \\
0 & -m a b
\end{array}\right] \text {, } \\
& \alpha=\frac{\theta_{1}^{-}-\theta_{2}^{-}}{2}
\end{aligned}
$$

where $\mathbf{Q}_{p}$ and $\mathbf{Q}_{m}$ represent transition matrices between swing and stance legs, + and - signs denote the state variables right after and right before the swing leg touchdown, respectively.
In this paper, we consider a minimalistic control strategy in which an open-loop motor controller plays an important role to induce self-stabilizing walking dynamics. The controller uses a sinusoidal oscillator with no sensory feedback. More specifically, torque of the hip motor $u_{H}$ is determined as follows:

$u_{H n}(t)=A_{n} \sin \left(2 \pi f_{n} t+\phi_{n-1}\right)$

where $A_{n}$ and $f_{n}$ are amplitude and frequency parameters at step $n$ that determine hip joint torque. Note that, in the rest of this paper, we consider an open-loop controller which varies the control parameters only at the end of every oscillation cycle. The variable $\phi_{n-1}$, therefore, represents the phase delays of the oscillator cycle at the moment of touchdown of the swing leg.

\subsection{Basin of attraction}

Basic locomotion stability of the compass gait model is shown in Fig. 2, which depicts projections of the return map. These figures illustrate all state variables of the model at the moments of touchdown while walking on a flat terrain with different oscillation of the hip motor explained above. A simulation result of passive walking on the level ground is shown in the left most plots, in which the model exhibits unsteady walking dynamics. More specifically, although stride length is decreased for the energy loss at every touchdown, all trajectories starting from three different initial conditions follow the fix points of state variables all the way until it falls over with the stride close to zero.

In contrast, the compass gait model exhibits a steady periodic locomotion with the energy input through the sinusoidal oscillation of hip motor. For example, Fig. 2 also shows three different frequency values of the hip oscillation, and the locomotion processes starting from different initial conditions converge to the same fix point and a constant stride length that is uniquely defined by the frequency parameter.

For more detailed analysis of the locomotion process, we investigate one step dynamics, which can be described as follows:

$$
\begin{aligned}
{\left[\begin{array}{c}
\mathbf{q}_{n+1}^{+} \\
\dot{\mathbf{q}}_{n+1}^{+} \\
\phi_{n+1}
\end{array}\right] } & =S\left(\mathbf{q}_{n}^{+}, \dot{\mathbf{q}}_{n}^{+}, \phi_{n}, f_{n}, A_{n}\right), \\
\phi_{n+1} & =\phi_{n}-\left(\frac{T_{n}}{2}-t_{T D}\right) \cdot 2 \pi f_{n}, \\
T_{n} & =\frac{1}{f_{n}}
\end{aligned}
$$

where the function $S$ computes the swing leg dynamics (1) and the collision dynamics (2), given $\mathbf{q}_{n}^{+}$and $\dot{\mathbf{q}}_{n}^{+}$representing the state variables right after the collision of the swing leg in step $n-1$. $t_{T D}$ indicates the duration between previ- 

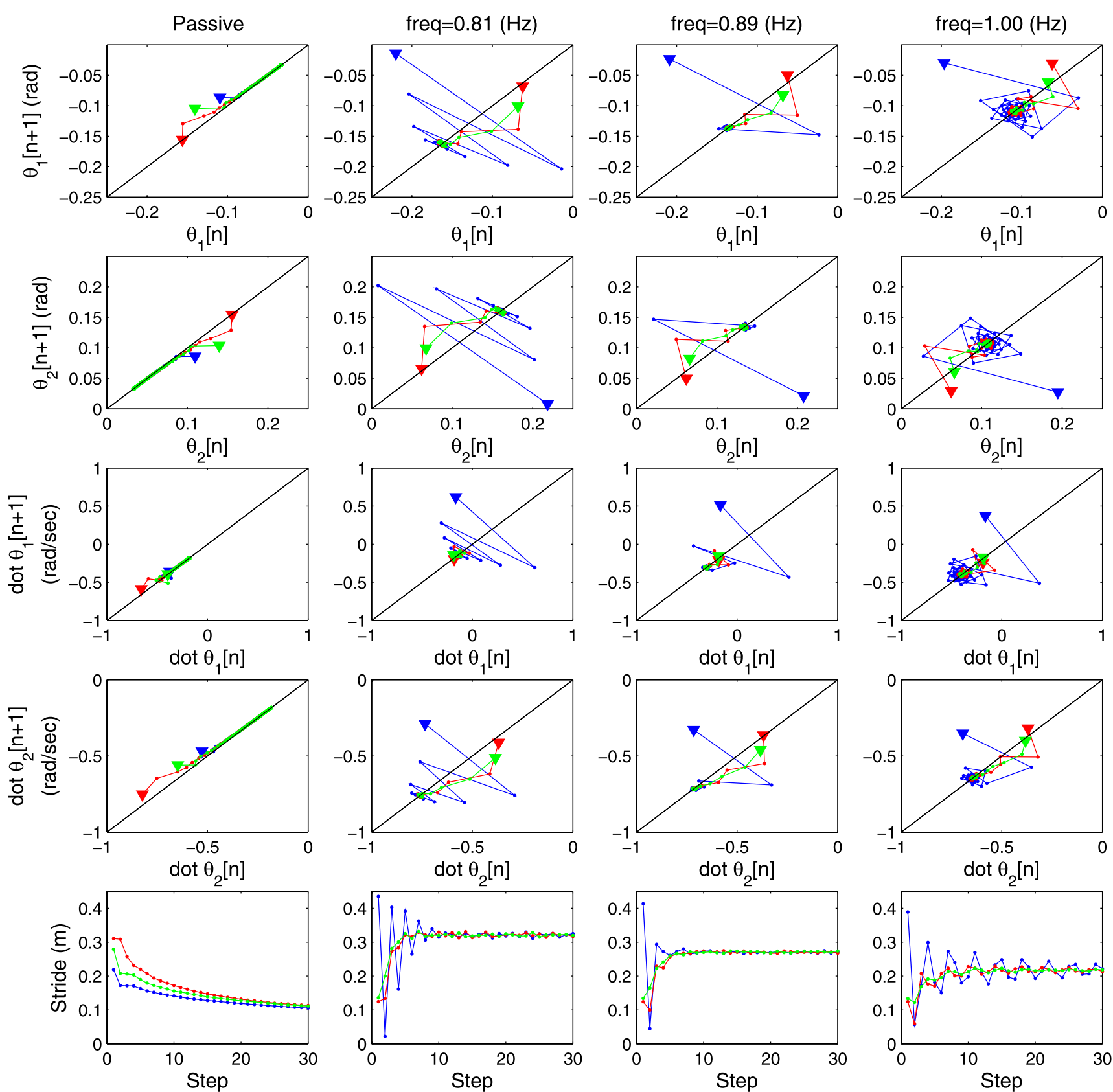

Fig. 2 Projections of the return map of the compass gait simulation with and without hip actuation. These projections depict the state variables $\left(\mathbf{q}=\left[\theta_{1}, \theta_{2}\right]^{T}\right.$ and $\dot{\mathbf{q}}=\left[\dot{\theta}_{1}, \dot{\theta}_{2}\right]^{T}$ at every touchdown of the swing leg, and three trajectories starting from different initial conditions (indicated by colored triangle plots) are shown in every diagram. Stride

ous and current collisions, and $A_{n}, f_{n}$ and $T_{n}$ are the amplitude, frequency, and period of hip motor oscillation, respectively (see (3)). A fix point can, therefore, be described as follows:

$\left[\begin{array}{c}\mathbf{q}^{*} \\ \dot{\mathbf{q}}^{*} \\ \phi^{*}\end{array}\right]=S\left(\mathbf{q}^{*}, \dot{\mathbf{q}}^{*}, \phi^{*}, f, A\right)$ length of every walking step is plotted in the lower figures, where stride length is decreased gradually without hip actuation while it converges to a certain value with the hip actuation. In these simulation experiments, the amplitude parameter is fixed at $A=1.0 \mathrm{~N} \mathrm{~m}$

Figure 3 (upper figures) shows how variations of initial phase delays $\phi_{0}$ converge to the phase delay at the fix point $\phi^{*}$, which essentially indicates the basin of attraction around the fix point. For example, with the frequency parameter $0.67 \mathrm{~Hz}$, the locomotion process starting from an initial phase delay $\phi_{0}=5.5 \mathrm{rad}$ converges to $\phi^{*}=2.9 \mathrm{rad}$ after approximately 15 steps. As shown in these figures, the basins of attraction are generally large enough that a signif- 

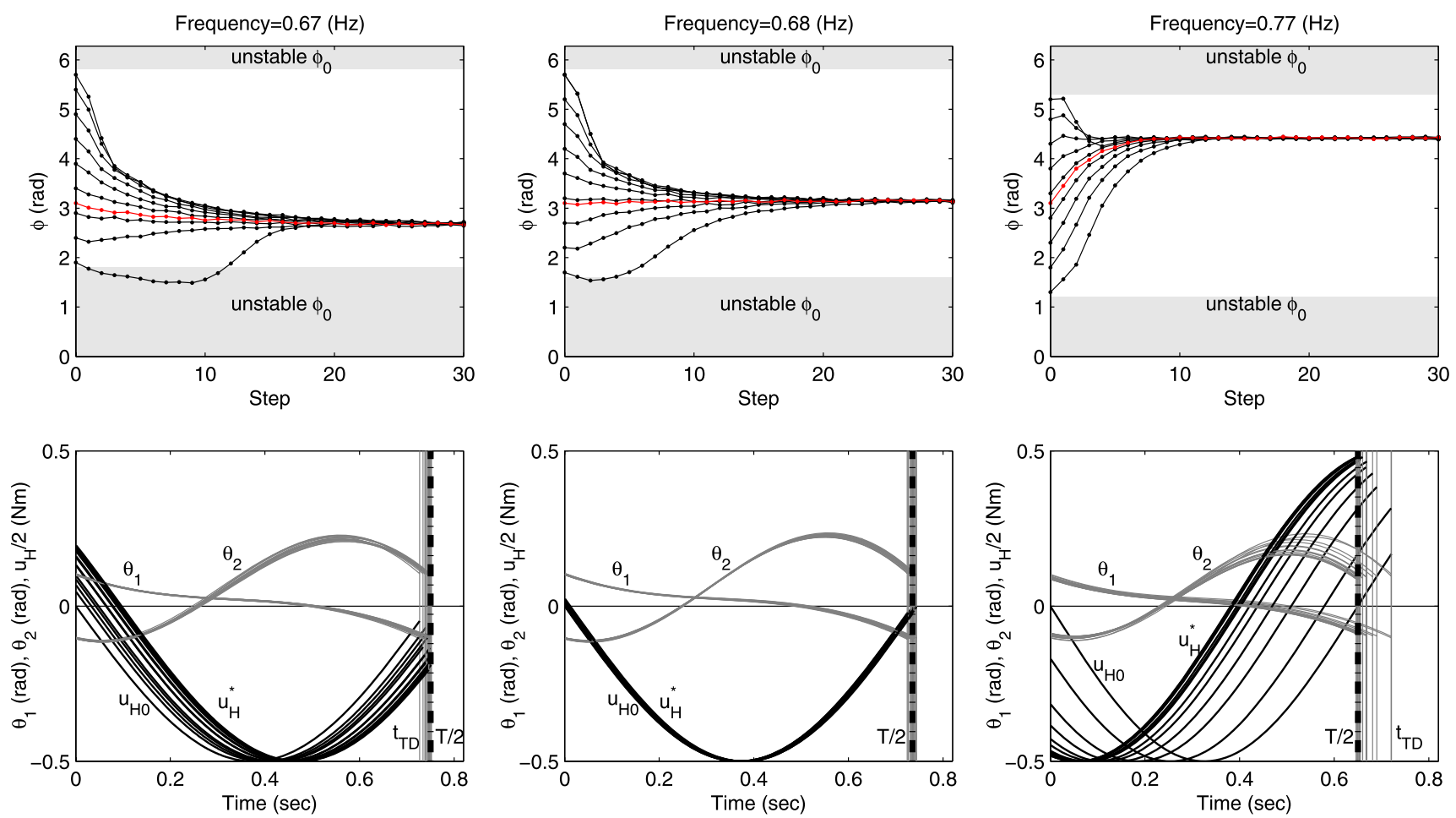

Fig. 3 The compass gait simulation with different frequency parameters. Upper figures show basins of attraction with respect to different initial phase delays $\phi_{0}$ in hip actuation. The unstable regions (shown in gray areas) represent the initial phase delays $\phi_{0}$ which do not converge to $\phi^{*}$ within 30 steps of locomotion. The detailed walking dynamics starting from $\phi_{0}=\pi$ (highlighted by the red lines) are shown in lower figures. These figures illustrate time series trajectories of state variables $\mathbf{q}$ (gray curves), hip motor torque $u_{H}$ (black curves), and the time of collision $t_{T D}$. Here the "phase locking" mechanism can be clearly observed by tracking a time of collision $t_{T D}$ converging to the period of motor oscillation $\frac{T}{2}$, particularly in left and right figures (i.e. the oscillation frequency $0.67 \mathrm{~Hz}$ and $0.77 \mathrm{~Hz}$, respectively). In these simulation experiments, the amplitude parameter is fixed at $A=1.0 \mathrm{~N} \mathrm{~m}$

\subsection{Variations of fix point}

More detailed trajectories of walking dynamics can be analyzed through the state variables and motor torque, which is also shown in Fig. 3 (lower figures). These figures illustrate the simulation started from an initial phase delay $\phi_{0}=\pi$ for all three frequency parameters. Here we clearly observe the "phase locking" mechanism, that is, a time of collision $t_{T D}$ converges to the period of motor oscillation $\frac{T}{2}$, and accordingly the phase delay $\phi$ (computed by (5)) converges to $\phi^{*}$.

It is important to note that, through these simulation experiments, we always found only one unique fix point represented by $\mathbf{q}^{*}, \dot{\mathbf{q}}^{*}$, and $\phi^{*}$ when the control parameters $f$ and $A$ are specified. Also another interesting characteristic shown in Fig. 3 (upper figures) is that it requires more steps to converge when an initial phase delay is smaller than the fix point, if compared with starting from larger ones. In addition, as a natural consequence of the phase locking mechanism, similar basins of attraction can also be observed when started from some deviations of the other initial parameters, i.e. $\mathbf{q}_{0}^{+}$and $\dot{\mathbf{q}}_{0}^{+}$.
So far we explained a basin of attraction induced by the phase locking mechanism, and how the variations of fixpoint walking dynamics can be generated by one of the model parameters (i.e. frequency parameter) through the same mechanism. The fix point, however, is not independently determined by a frequency parameter, but strongly coupled with the other model parameters including the amplitude of oscillator $A$ and the slope angle $\gamma$, for example. The goal of this section, therefore, is to characterize the influence of model parameters in relation to the phase locking mechanism, and to explore possible walking dynamics induced by the proposed control approach.

The first set of simulation was conducted on a flat ground $\gamma=0 \mathrm{rad}$, and we searched fix points with respect to both control parameters $A$ and $f$. As explained in the previous subsection, each fix point (represented by $\mathbf{q}^{*}, \dot{\mathbf{q}}^{*}, \phi^{*}$ ) can be uniquely found once we set these control parameters, and the result is shown in Fig. 4. Note that, once a fix point is found, we are also able to estimate stride length of the fix point Stride ${ }^{*}$, which is an important metric to determine footholds during locomotion in rough terrain. From the state variable 

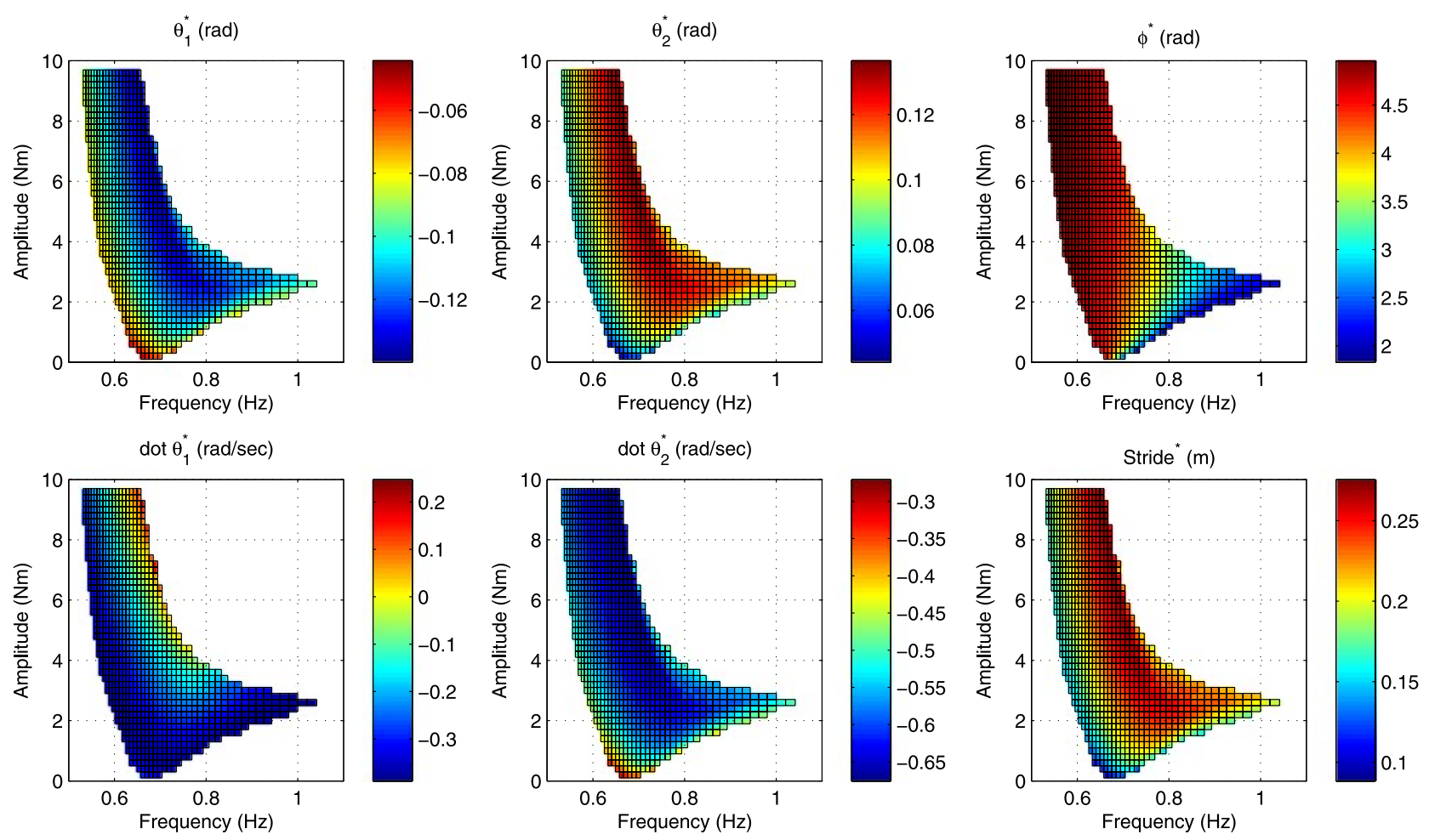

Fig. 4 State variables, phase delays, and stride lengths at fix points on a flat terrain with respect to the control parameters $A$ and $f$. Stride* is calculated based on $\mathbf{q}^{*}=\left[\theta_{1}^{*}, \theta_{2}^{*}\right]^{T}$ and the leg length $l$ as explained in (8)

$\mathbf{q}^{*}=\left[\theta_{1}^{*}, \theta_{2}^{*}\right]^{T}$, Stride $e^{*}$ can be estimated as follows:

$$
\text { Stride }^{*}=l\left(\sin \theta_{1}^{*}+\sin \theta_{2}^{*}\right)
$$

In general, from the figure of Stride* (lower right figure in Fig. 4), it is shown that stride length generated by the open-loop controller is essentially influenced not only by the frequency parameter $f$ but also by the amplitude parameter $A$. In particular, around the parameter space $f \simeq 0.7 \mathrm{~Hz}$ and $0.0<A<6.0 \mathrm{~N} \mathrm{~m}$, large variations of stride length can be achieved with respect to the amplitude parameter. With smaller values of the frequency parameter (e.g. $0.5<f<$ $0.6 \mathrm{~Hz}$ ), however, we cannot expect significant variations of stride length even by large changes of the amplitude parameter. In contrast, regardless of the amplitude value, it is possible to control stride length approximately between 0.15 and $0.25 \mathrm{~m}$ when the frequency parameter is varied.

It is also shown that, from the figure of phase delay (upper right plot in Fig. 4), the phase delays between mechanical dynamics and the oscillator is more significant with respect to the frequency parameter if compared with the amplitude parameter (especially at a smaller amplitude parameter, i.e. $A \simeq 2.5 \mathrm{~N} \mathrm{~m}$ ). This essentially means that, when the robot varies the frequency parameter at a smaller amplitude parameter for a switch of stride length, it requires many leg steps for the transition between one stride length to the other.
The fix points can be also found in locomotion on inclined slopes, and Fig. 5 shows stride length Stride* with respect to the frequency parameter in various slopes (the amplitude parameter is fixed at $A=7.0 \mathrm{Nm}$ ). In general, it is possible to control stride length also in inclined slopes through the frequency parameter by considering the fact that stride length becomes smaller as the frequency parameter increases. However, it is generally the case that control of shorter stride is more difficult in downhill slopes $(\gamma<0)$, and longer one in uphill $(\gamma>0)$. Moreover, variations of stride lengths tend to be richer in uphill locomotion since Stride ${ }^{*}$ exists between 0.10 and $0.25 \mathrm{~m}$ in the slope angle $\gamma=0.005 \mathrm{rad}$, whereas it is much narrower in downhill slopes (e.g. $0.30<$ Stride $^{*}<0.34 \mathrm{~m}$ in the slope $\gamma=-0.015 \mathrm{rad})$. Note that, while the walking dynamics in the inclined slopes are also dependent on the amplitude parameter $A$, we found that the characteristics explained here (i.e. the relation between stride length, inclination of slopes, and the frequency parameter) are preserved over a large variety of the parameter.

\section{Dynamics of a compass gait robot}

For a real-world evaluation of the proposed control framework, we developed a robot platform based on the compass 
Fig. 5 Stride length at the fix points determined by frequency parameter $f$ and slope angles $\gamma$ $(\gamma<0$ indicates downhill slopes, and $\gamma>0$ uphill slopes). Amplitude parameter is fixed at $A=7.0 \mathrm{Nm}$ in these

experiments. In general, a larger amplitude value results in more variations of walking dynamics in inclined slopes

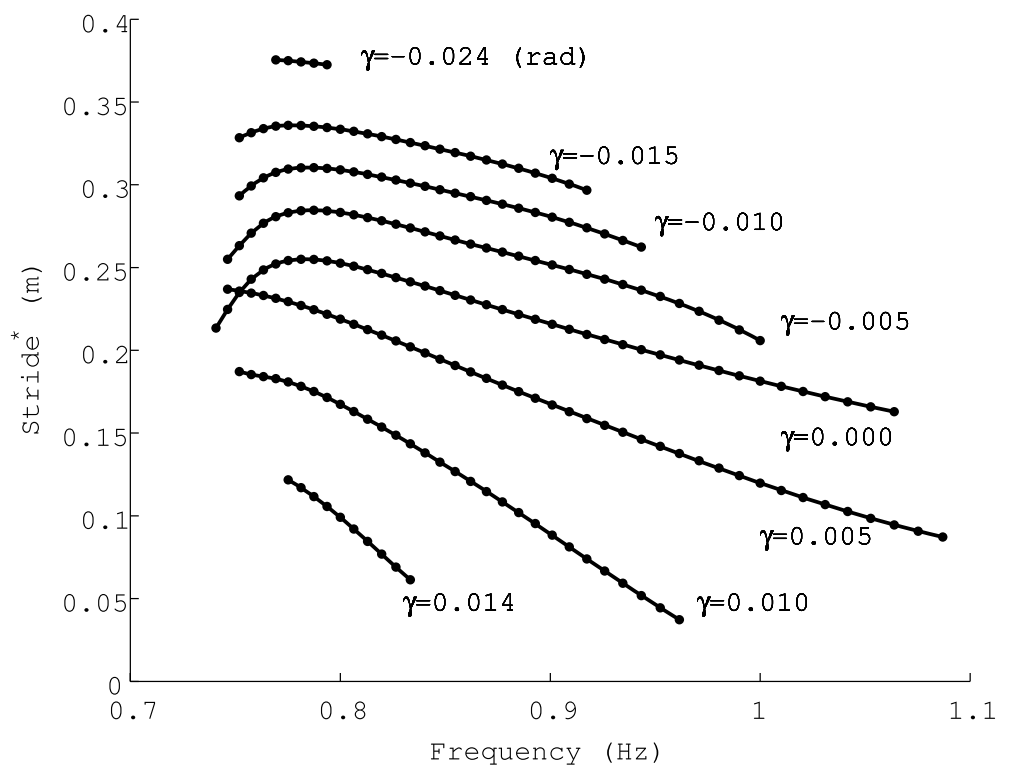

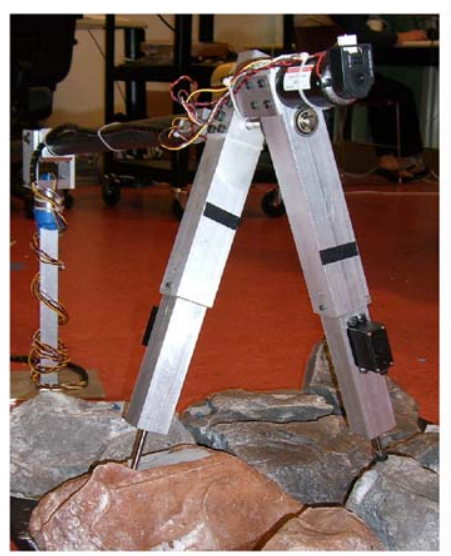

(a)

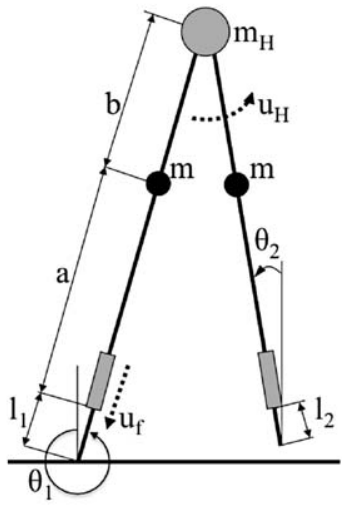

(b)
Fig. 6 (a) Photograph of compass gait robot, and (b) Compass gait model with hip and foot actuators (gray circle and rectangles)

gait model with a few practical modifications. In this section, we first describe the design and control of the platform, then behavioral characteristics are analyzed through locomotion experiments.

\subsection{Design and control of robot}

The robot platform shown in Fig. 6. consists of two leg segments connected through a hip joint, where a direct-drive motor (Maxon Motor RE40 with no gear reduction) exerts torque between two legs. The hip joint is then connected to a boom that allows pitch rotation while restricting yaw and roll. At the other end of the boom, we installed a counter weight to avoid a large ground impact of every step, and of harmful crashes of the entire robot (see Table 2 for more specifications of the robot platform).

\begin{tabular}{lll}
\hline Symbol & Description & Value \\
\hline$a$ & Lower leg segments & $0.260 \mathrm{~m}$ \\
$b$ & Upper leg segments & $0.055 \mathrm{~m}$ \\
$l_{1}, l_{2}$ & Foot segments & $0.000-0.040 \mathrm{~m}$ \\
$m$ & Mass of leg & $1.3 \mathrm{~kg}$ \\
$m_{H}$ & Mass of body & $0.2 \mathrm{~kg}$ \\
$C W$ & Counter weight & $4.1 \mathrm{~kg}$ \\
$B L 1$ & Boom length to robot & $1.210 \mathrm{~m}$ \\
$B L 2$ & Boom length to counter weight & $0.560 \mathrm{~m}$ \\
$A$ & Amplitude of oscillation & $1.0 \mathrm{~N} \mathrm{~m}$ \\
$P_{i, 12}$ & Amplitude of foot extension & $0.000-0.015 \mathrm{~m}$ \\
$\psi$ & Phase delay of foot oscillation & $2.2 \mathrm{rad}$ \\
\hline
\end{tabular}

In contrast to the simulation model, foot retraction is necessary to avoid the swing leg colliding with the ground, and for this reason, each leg segment has a servomotor (Hitech HSR-5980SG) that extends and retracts a foot segment for ground clearance during swing phase. To reduce the difference in dynamics between the simulation and the real-world experiments, we minimize the mass of the foot segments such that they are negligible. Because of the foot actuation, the state variables of this platform are $\mathbf{q}=\left[\theta_{1}, \theta_{2}, l_{1}, l_{2}\right]^{T}$ and their velocity components $\dot{\mathbf{q}}$. In addition to the sinusoidal oscillation of hip motor torque described by (3), the robot receives an additional control input for control of foot actuators. The motor torque $u_{f i}$ of the foot motor $i$ can be described as follows:

$u_{f i}(t)=K_{p}\left(l_{i}-P_{i}(t)\right)+K_{d}\left(i_{i}-0.0\right)$,
Table 2 Specification of robot 
Fig. 7 Basin of attraction with and without hip actuation. Phase plots (top), return maps of the robot's outer leg (middle), and corresponding stride length (bottom). Red triangles denotes the beginning of data recording. In both experiments, the oscillation frequency is set to $1.0 \mathrm{~Hz}$
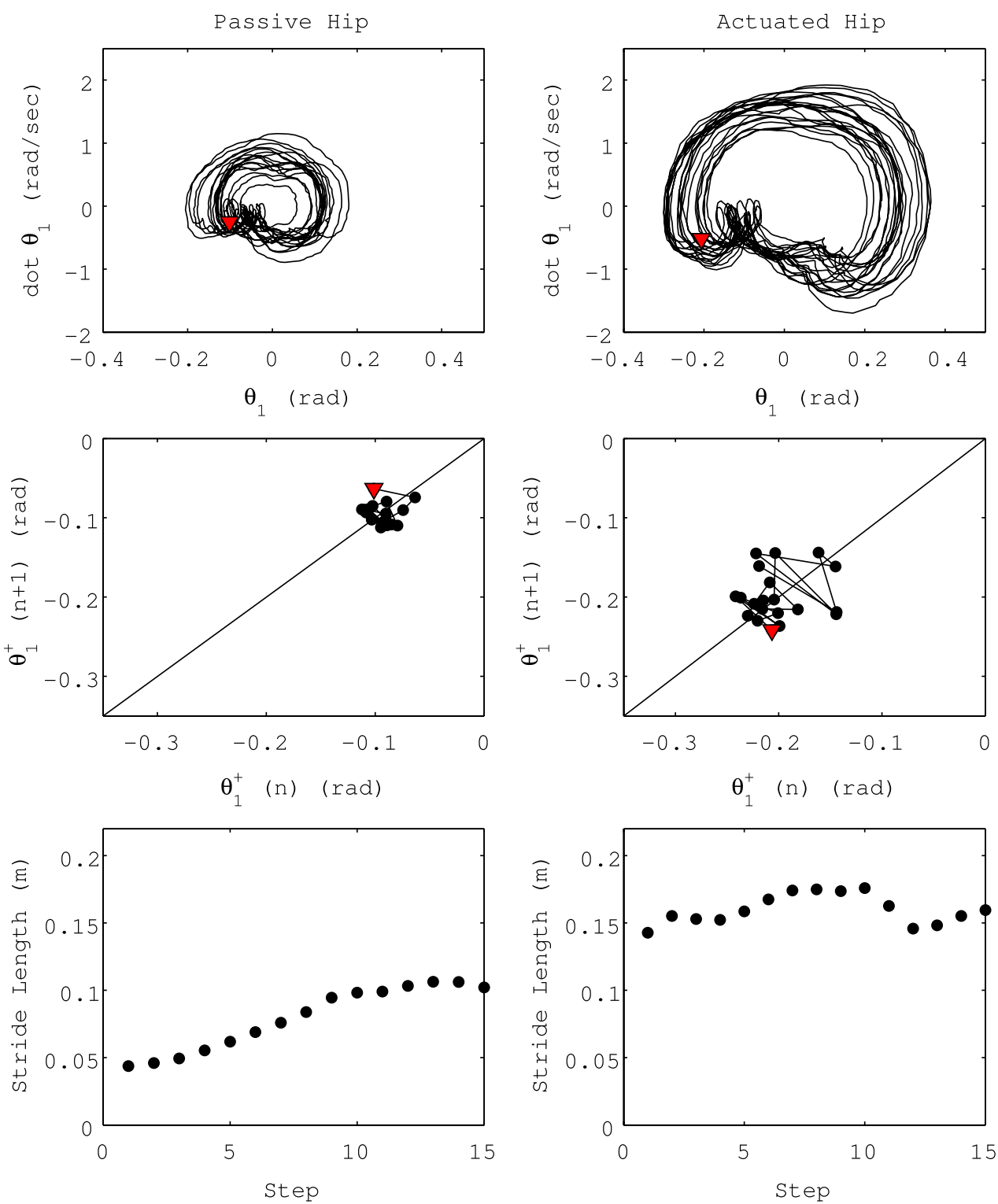

$P_{i}(t)=\left\{\begin{array}{l}P_{i 1}: \sin (2 \pi f t+\psi)>0 \\ P_{i 2}: \text { otherwise }\end{array}\right.$

$$
(i=1,2)
$$

where $K_{p}$ and $K_{d}$ are the proportional and differential gains of PD controller, and $P_{i\{1,2\}}$ represents the given setpoints of the foot segment $i$.

For sensory feedback and measurement of locomotion dynamics, we implemented an encoder at the hip motor (Maxon Motor HEDS5540), force sensitive resistors in both foot segments, and a potentiometer that measures horizontal position around the boom. These motors and sensors are connected to a PC104 computer (Digital-Logic MSMP5SEN) with a sensor board (Sensoray Model-526), which enables the control bandwidth of approximately $100 \mathrm{~Hz}$. In addition, in order to measure the overall dynamics of the robot during locomotion, we conducted the experiments under the motion capture systems (Vicon MX consisting of 16 cameras, which use infrared light to track reflective markers on the robot at approximately $120 \mathrm{~Hz}$ sampling rate).

\subsection{Steady state dynamics}

When we properly set the control parameters described in the previous section, the compass gait robot exhibits stable periodic walking gait on a flat terrain. In order to characterize basic behaviors of the robotic platform, the first set of experiments were conducted on a flat terrain with a few different configurations of control parameters.

Figure 7 shows a phase plot and return map of a leg, and stride length of every step with and without the hip motor control. As shown in the left plots of Fig. 7, the basic locomotion dynamics of the compass gait robot can be generated 
simply by using the foot segment control without hip actuation (i.e. $u_{H}=0.0$ ). Specifically, even when starting with an initial condition $\left[\theta_{1}, \theta_{2}, \dot{\theta}_{1}, \dot{\theta}_{2}\right]^{T}=[0,0,0,0]^{T}$, walking dynamics of the robot reaches a relatively stable walking dynamics after several steps. This behavioral characteristics in the robotic platform is clearly different from those of the simulation model, which is essentially induced by the actuation of foot segments. It is also important to note that, while this walking dynamics is seemingly stable on a flat terrain, the walking direction is not controllable (the limit cycle of forward or backward walking is largely dependent on the initial conditions and environment), and the robot is not able to walk uphill. In contrast, with the hip actuation (Fig. 7 right plots), we can observe a similar limit cycle of locomotion, but the amplitude and the perturbation of leg swing are much larger resulting in the longer stride.

Figure 8 shows trajectories of the motor command $\left[u_{H}, u_{f 1}\right]^{T}$ and the state variables $\mathbf{q}=\left[\theta_{1}, \theta_{2}\right]^{T}$ of successive ten steps, which are aligned with respect to the ground contact detected by the foot pressure sensors. This figure shows the phase locking in the real-world platform, which can be observed by how the hip motor oscillation is synchronized with the mechanical dynamics regardless of the different oscillation frequencies. In addition, it is important to note that another common characteristics of the simulation model and the robot lies in the amplitude of swing legs, which decreases as the frequency parameter increases (this feature can also be observed in Fig. 3, lower figures).

\subsection{Gait variability}

As in the simulation analysis, we also conducted a set of experiments to examine stride length with respect to the frequency parameter of the oscillator and the slope angles. Figure 9 (the filled circle plots) shows the mean stride length and standard deviation of ten steps of walking with respect to the set of frequency parameter. From this figure, we can see that it is possible to increase stride length of the steady state locomotion approximately $50 \%$ by changing the frequency from 1.11 to $0.77 \mathrm{~Hz}$.

The same set of frequency parameters was also tested in different inclinations of slopes in order to analyze controllability of foot placement in rough terrains. Figure 9 shows that the robot is able to walk with different stride length in large variety of slopes (between +0.087 and $-0.122 \mathrm{rad}$ ). On the level ground, the variability of stride length is between approximately 0.09 and $0.15 \mathrm{~m}$. With the same range of the frequency parameter, the stride length becomes larger in downhill, and smaller in uphill environments. An important characteristic of the proposed control architecture is the fact that the robot is not able to walk uphill with the lower frequency of oscillator, and downhill with the higher frequency. More specifically, the +0.087 radian uphill can be dealt with by the frequency of $0.83 \mathrm{~Hz}$ and larger, and the robot can walk down -0.122 radian slope with the frequency of $1.0 \mathrm{~Hz}$ or lower. In other words, the limit of the proposed controller is the control of small stride in downhill, and large stride in uphill.

\section{Locomotion control in rough terrain}

One of the most significant advantages of the proposed control architecture lies in the fact that, by exploiting the selfstability, one control parameter is sufficient to vary the basic walking dynamics. This section explains how the aforementioned open-loop controller can be extended with a sensory feedback to deal with a rough terrain, and analyzes the locomotion performance in complex environment.

\subsection{Feedback controller}

For the sake of simplicity, we assume that the feedback controller receives only the location of horizontal axis every control step, and determines the frequency value of the oscillator. The feedback controller, therefore, can be described as

$f=\operatorname{freq}(x, t)$

where $x$ represents the current horizontal position of the hip joint with respect to an absolute coordinate system. The function freq $(x, t)$ also depends on the time variable of oscillator, because the controller is allowed to change the frequency only at the end of every oscillation period for smooth transitions of motor command.

In the following case study, we heuristically determined the function $f r e q(x, t)$ for a given rough terrain. Owing to the minimalistic control architecture, it requires only several trials and errors until we found a set of thresholds for the parameter $x$ for multiple successful travels over the rough terrain.

\subsection{Experiments}

In this case study, we tested the proposed controller in a test environment consisting of a flat terrain, an uphill slope, molded "flat rocks", and a downhill as shown in Figs. 10 and 11. The important features of this terrain are a +0.065 radian uphill, -0.045 radian downhill, $0.60 \mathrm{~m}$ of rough terrain with the largest gap length of $0.03 \mathrm{~m}$ and the largest step hight of $+0.02 \mathrm{~m}$ and $-0.03 \mathrm{~m}$.

After several trials and errors, we set the control parameters as follows:

freq $(x, t)=\left\{\begin{array}{l}0.77 \mathrm{~Hz}: x<3.4 \mathrm{~m} \\ 1.11 \mathrm{~Hz}: x \geq 3.4 \text { and } x<4.5 \mathrm{~m} \\ 1.00 \mathrm{~Hz}: x \geq 4.5 \text { and } x<5.1 \mathrm{~m} \\ 0.77 \mathrm{~Hz}: x \geq 5.1 \mathrm{~m}\end{array}\right.$ 

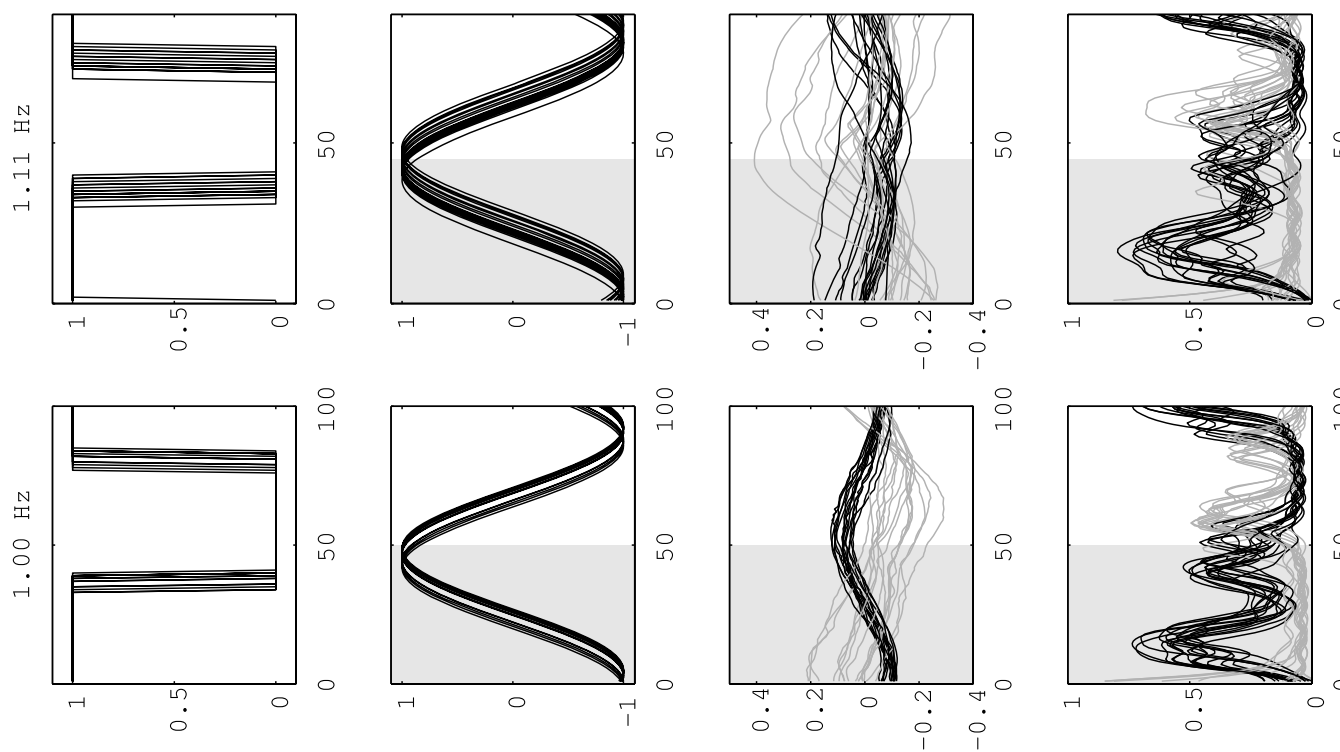

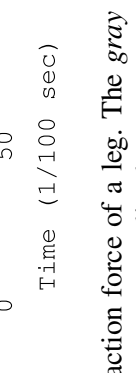
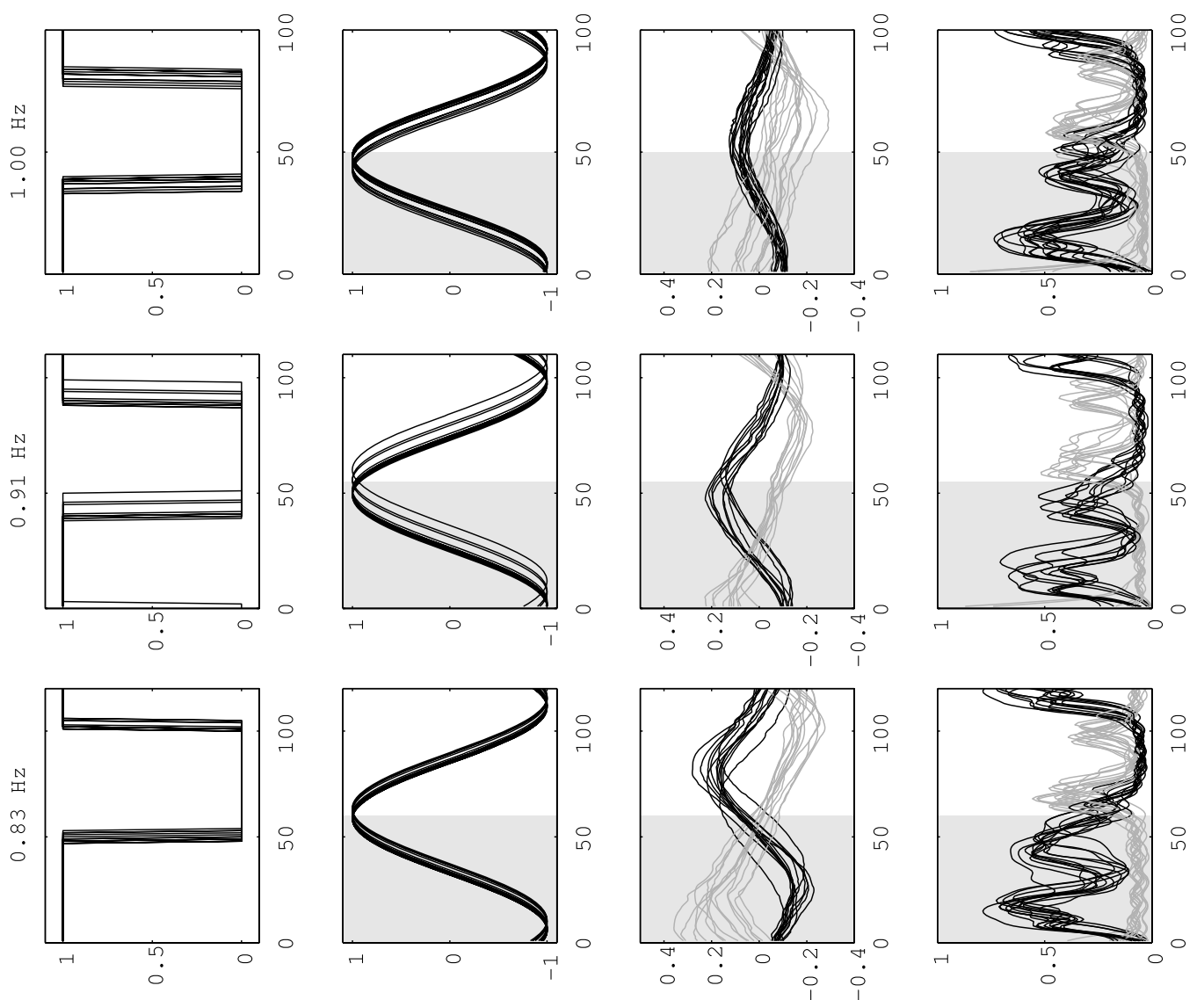

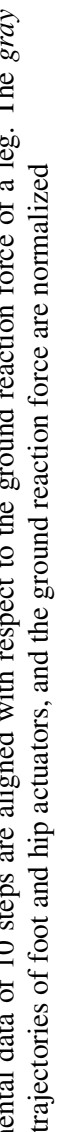
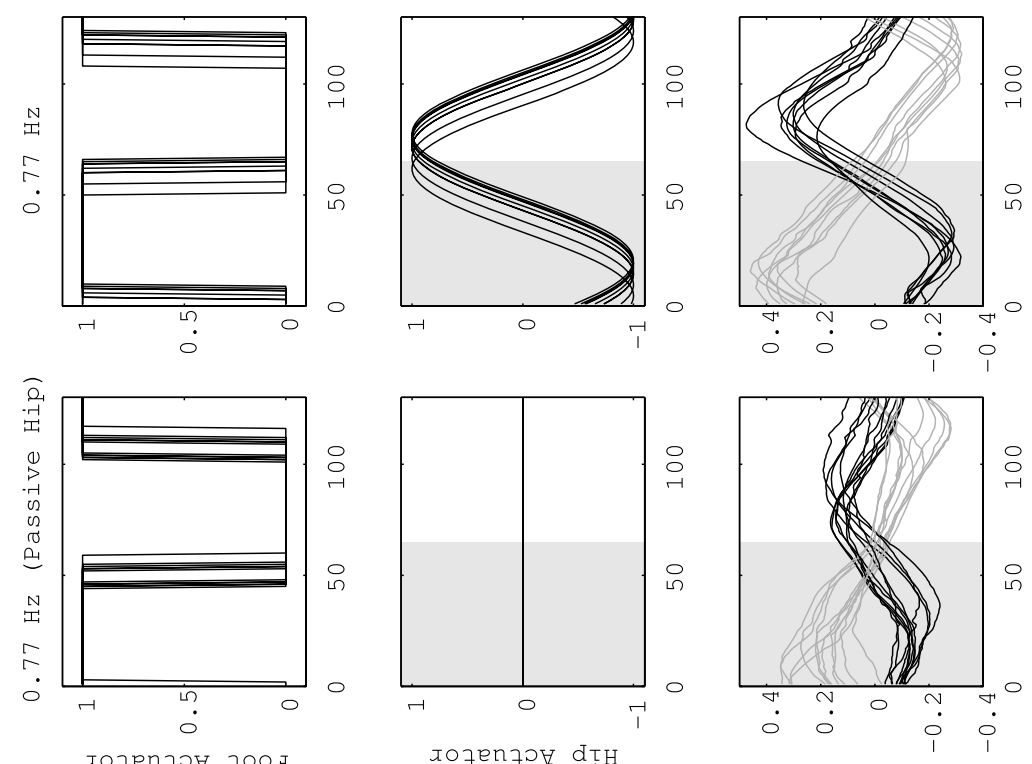

(pex) sәtбut 6әт

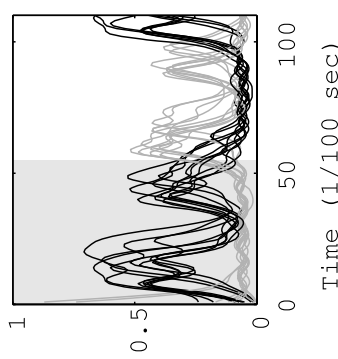

焉

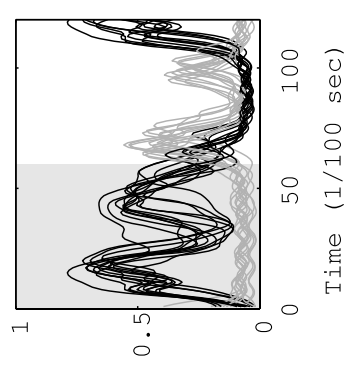

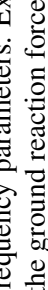

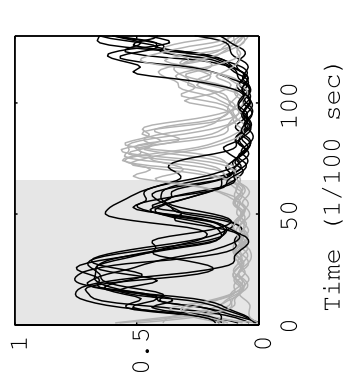

동

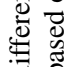

象

के

突㻤

on

30

ло7еn7ग 700

лоาеกาว dṬH

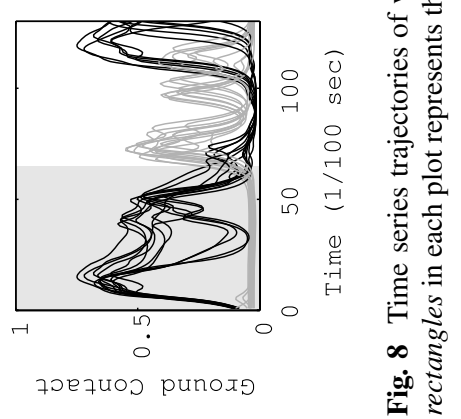




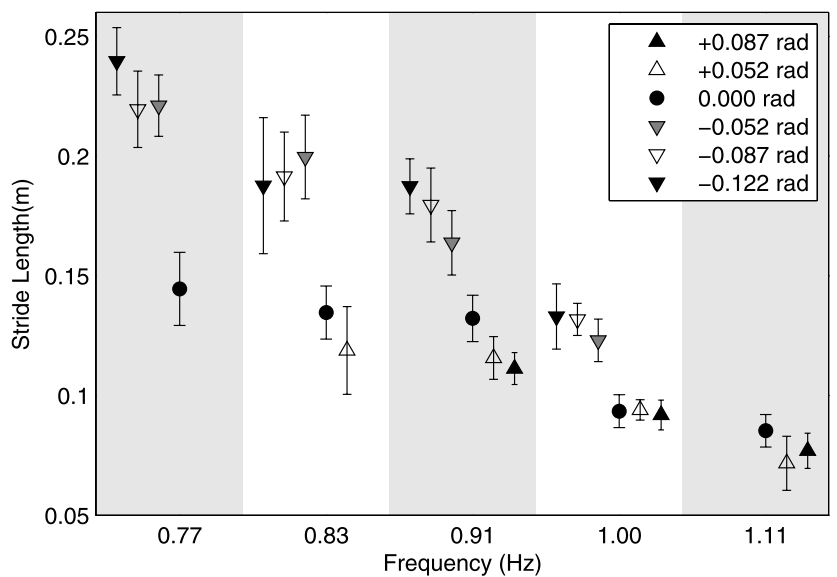

Fig. 9 Variability of stride with respect to five frequency parameters in the different inclinations of slope (downhill $-0.052,-0.087$, $-0.122 \mathrm{rad}$, level ground, and uphill: +0.052 and $+0.087 \mathrm{rad})$. Every plot represents a mean stride length of 10 steps and their standard deviation in each environment

Based on the basic knowledge about the gait variability (shown in Fig. 9), here we set the frequency parameter to a larger value for the uphill slope, and set to a smaller frequency for larger strides in the downhill slope and flat surfaces. This controller was tested under the motion capture system which recorded the kinematics of the robot as shown in Fig. 10, and the kinematic data of three successful travels over the rough terrain are reproduced in Fig. 11.

In general, the controller is able to maintain the locomotion mostly on the flat surfaces including the uphill, the downhill, and the small step around $x=3.6 \mathrm{~m}$. Moreover the controller also is able to cope with locomotion over sparse gaps and steps on the ground by appropriately setting the function $f r e q(x, t)$, even though there are some variance in the foot placement. It is important to note that the online modification of frequency parameter essentially requires a few steps of transient period before converging to a steady stride length shown in Fig. 9. In Fig. 11, for example, the stride length changed from $0.19 \mathrm{~m}$ to $0.05 \mathrm{~m}$ (at around $x=3.5 \mathrm{~m}$ ), but approximately three steps were necessary for the convergence. In these experiments, therefore, we needed several trials and errors to determine $\operatorname{freq}(x, t)$ in (12). In addition, another potential limitation of the controller is that it occasionally failed on the rocks $(x=4.9-$ $5.5 \mathrm{~m})$. Considering the large variance of stride length in this area of the terrain shown in Fig. 11, the main reason of failures seems to be originated in the irregular ground interactions.

\section{Conclusion}

This paper presented a minimalistic control architecture for dynamic walking of the compass gait model. The controller

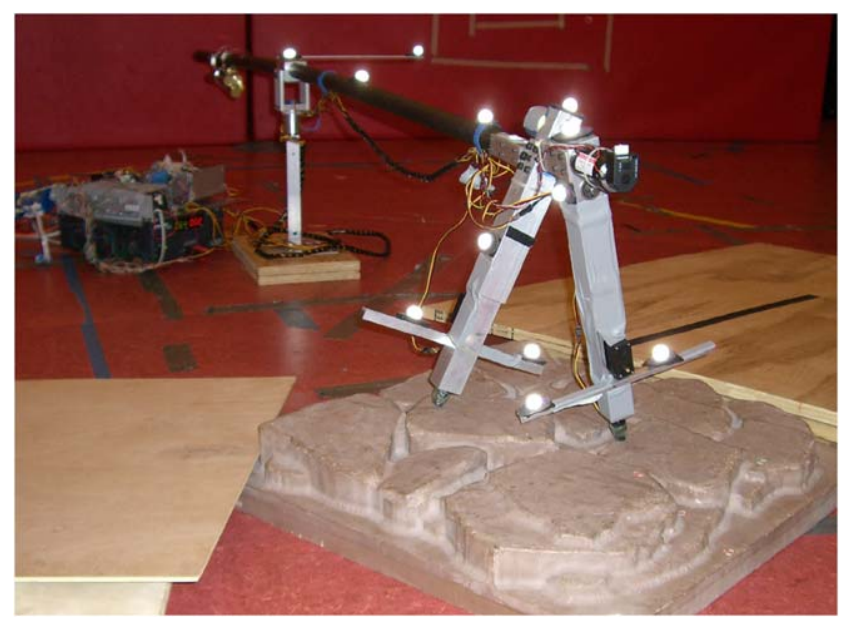

Fig. 10 Rough terrain experiment with the motion capture system. 12 markers are attached in the boom and legs

makes use of an open-loop sinusoidal oscillation at the hip joint, and we identified the phase locking mechanism that self-calibrates phase delays between walking dynamics and oscillation of the hip motor. This mechanism can be nicely explained by a fix point analysis, by which we could also systematically investigate the relation between walking dynamics and the motor control parameters. The main contribution of this paper lies in the fact that, owing to the phase locking mechanism, a simple open-loop based controller can deal with various uneven terrains such as steady walking in uphill and downhill slopes as well as controlling foot placement to deal with gaps and steps. This minimalistic controller is particularly important for planning and optimization of locomotion control in moderately complex environment. In the case study we showed in Sect. 4, for example, it required only several trials and errors until we found the set of parameters. It should, therefore, be straight forward to automate the search process of control parameters by using a depth-first algorithm, for example.

The self-stability of dynamic walking achieved by the proposed control architecture is comparable to those of the bio-inspired oscillators, typically labeled as the central pattern generator (CPG) models. Although both approaches induce the synergy between motor oscillation and mechanical dynamics for a stable periodic locomotion in a selforganized manner, the present work demonstrated that a set of relatively large basins of attraction can be achieved without explicit sensory feedback. In addition, owing to its simplicity, we were able to conduct a systematic analysis of behavioral variations as well as an extended control architecture for foot placement in complex rough terrain. It is, however, still an open question to what extent the locomotion performance (e.g. stability and controllability of walking dynamics) is different in these two approaches. From this perspective, it is particularly interesting to conduct a compara- 

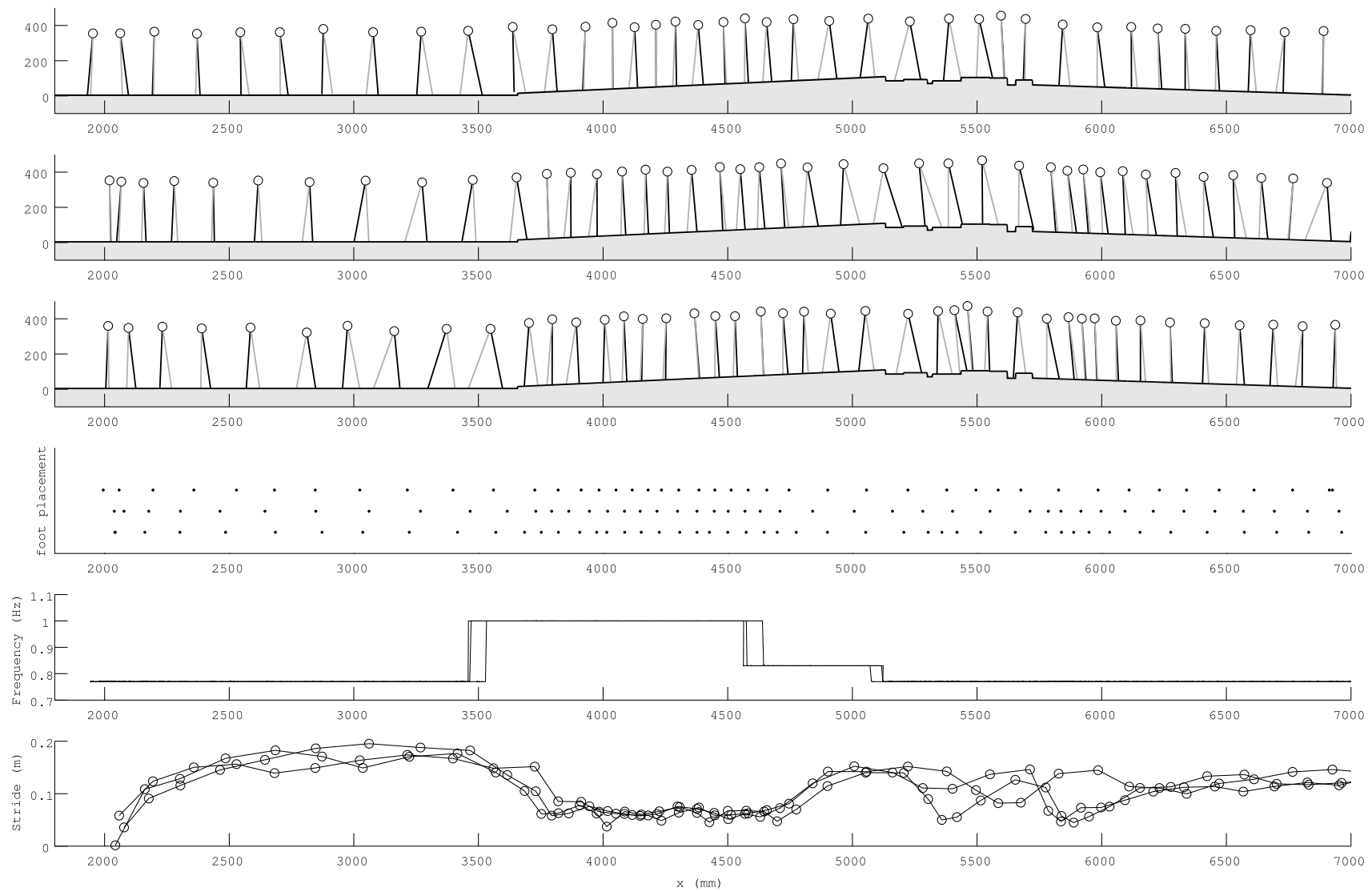

Fig. 11 Walking experiments in rough terrain. Top figures show the trajectories of the three successful travels of the rough terrain. The location and pose of the robot are depicted every 0.8 second. The dots in the middle plot represent foot placement over three successful trials. The lower plots show the frequency parameter of oscillator, and the stride length of every step during the three successful trials tive study between the proposed controller and the other approaches such as the phase resetting controllers, the reflexbased controllers and the CPG-based controllers (Aoi and Tsuchiya 2007; Taga et al. 1991; Manoonpong et al. 2007; Ijspeert 2008).

For dynamic locomotion in more complex environment, however, we also identified a few potential limitations of the proposed control framework. First, as shown in the simulation of Sect. 2.3 and the real-world experiment of Sect. 3.2, controllability of stride length is degraded as the angle of slope increases for both uphill and downhill. Second, we still do not know the influence of the impact force at ground contact and the counterweight to the stability and walking dynamics in the real-world experiments. For example, there should be a control architecture of foot actuation that improves locomotion performance (as exemplified in Byl and Tedrake (2008a, 2008b)), which we have not considered in details in this article. And third, in the proposed control approach, it requires several steps until a stride length converges to another when switching the control parameter of the oscillator. For example, in the upper plots of Fig. 3, it took approximately three to ten steps until it converges to a steady stride length when the simulated compass gait model started with various initial conditions. And in Fig. 11, we also observed three to five steps of transition steps when the controller switched the parameter in the real-world platform.

To cope with these open problems, we expect two future research directions based on our achievement presented in this paper. One of the potential extensions of the proposed controller is to examine the effects of different oscillator trajectories. For example, although we tested only control of frequency parameter in this paper, the amplitude parameter of the oscillator could potentially provide an additional increase of controllability as our simulation analysis suggested in Sect. 2.3. Second, it is also important to pursue the use of sensory feedback in the low-level controller. In particular, it is interesting to investigate further how the phase locking mechanism identified in this paper can be integrated into a more comprehensive optimization process of statefeedback controllers as demonstrated in Byl and Tedrake (2008a, 2008b), Manchester et al. (2009), for example.

Acknowledgements This work is supported by the National Science Foundation (Grant No. 0746194) and the Swiss National Science Foundation (Grant No. PBZH2-114461 and PP00P2_123387/1). 


\section{References}

Adamczyk, P. G., Collins, S. H., \& Kuo, A. D. (2006). The advantages of a rolling foot in human walking. Journal of Experimental Biology, 209, 3953-3963.

Aoi, S., \& Tsuchiya, K. (2005). Locomotion control of biped robot using nonlinear oscillators. Autonomous Robots, 19, 219-232.

Aoi, S., \& Tsuchiya, K. (2006). Stability analysis of a simple walking model driven by an oscillator with a phase reset using sensory feedback. IEEE Transactions on Robotics, 22(2), 391-397.

Aoi, S., \& Tsuchiya, K. (2007). Self-stability of a simple walking model driven by a rhythmic signal. Nonlinear Dynamics, 48(1), $1-16$.

Asano, F., Yamakita, M., \& Furuta, K. (2000). Virtual passive dynamic walking and energy-based control laws. In IEEE/RSJ international conference on intelligent robots and systems (IROS 2000) (pp. 1149-1154).

Asano, F., Yamakita, M., Kamamichi, N., \& Luo, Z.-W. (2004). A novel gait generation for biped walking robots based on mechanical energy constraint. IEEE Transactions on Robotics and Automation, 20(3), 565-573.

Asano, F., Hayashi, T., Luo, Z. W., Hirano, S., \& Kato, A. (2007). Parametric excitation approaches to efficient dynamic bipedal walking. In Proc. of the IEEE/RSJ int. conf. on intelligent robots and systems (pp. 2210-2216).

Byl, K., \& Tedrake, R. (2008a). Approximate optimal control of the compass gait on rough terrain. In Proceedings IEEE international conference on robotics and automation (ICRA) (pp. 1258-1263).

Byl, K., \& Tedrake, R. (2008b). Metastable walking machines. International Journal of Robotics Research, 28(8), 1040-1064.

Collins, S. H., Wisse, M., \& Ruina, A. (2001). A three-dimensional passive-dynamic walking robot with two legs and knees. International Journal of Robotics Research, 20, 607-615.

Collins, S. H., Ruina, A., Tedrake, R., \& Wisse, M. (2005). Efficient bipedal robots based on passive-dynamic walkers. Science, 307, 1082-1085.

Garcia, M., Chatterjee, A., Ruina, A., \& Coleman, M. (1998). The simplest walking model: stability, complexity, and scaling. Journal of Biomechanical Engineering. Transactions of the ASME, 120(2), 281-288.

Goswami, A., Espiau, B., \& Keramane, A. (1997). Limit cycles in a passive compass gait biped and passivity-mimicking control laws. Autonomous Robots, 4, 273-286.

Goswami, A., Thuilot, B., \& Espiau, B. (1998). A study of the passive gait of a compass-like biped robot: symmetry and chaos. International Journal of Robotics Research, 17(12), 1282-1301.

Harata, Y., Asano, F., Luo, Z. W., Taji, K., \& Uno, Y. (2007). Biped gait generation based on parametric excitation by knee-joint actuation. In Proc. of the IEEE/RSJ int. conf. on intelligent robots and systems (pp. 2198-2203).

Hass, J., Herrmann, J. M., \& Geisel, T. (2006). Optimal mass distribution for passivity-based bipedal robots. International Journal of Robotics Research, 25(11), 1087-1098.

Hobbelen, D. G. E., \& Wisse, M. (2008). Swing-leg retraction for limit cycle walkers improves disturbance rejection. IEEE Transactions on Robotics, 24(2), 377-389.

Iida, F., \& Tedrake, R. (2009). Minimalistic control of a compass gait robot in rough terrain. In International conference on robotics and automation (ICRA 09) (pp. 1985-1990).

Iida, F., Rummel, J., \& Seyfarth, A. (2008). Bipedal walking and running with spring-like biarticular muscles. Journal of Biomechanics, 41, 656-667.

Ijspeert, A. J. (2008). Central pattern generators for locomotion control in animals and robots: a review. Neural Networks, 21(4), 642653.
Kajita, S., \& Espiau, B. (2008). Legged robots. In B. Siciliano \& O. Khatib (Eds.), Springer handbook of robotics (pp. 361-389). Berlin: Springer.

Kim, J., Choi, C., \& Spong, M. (2007). Passive dynamic walking with symmetric fixed flat feet. In International conference on control and automation (pp. 24-30).

Kinugasa, T., Miwa, S., \& Yoshida, K. (2008). Frequency analysis for biped walking via leg length variation. Robotics and Mechatronics, 20(1), 98-104.

Kuo, A. D. (1999). Stabilization of lateral motion in passive dynamic walking. International Journal of Robotics Research, 18(9), $917-$ 930.

Kuo, A. D. (2002). Energetics of actively powered locomotion using the simplest walking model. Journal of Biomechanical Engineering, 124, 113-120.

Kurz, M. J., \& Stergiou, N. (2005). An artificial neural network that utilizes hip joint actuations to control bifurcations and chaos in a passive dynamic bipedal walking model. Biological Cybernetics, 93, 213-221.

Kwan, M., \& Hubbard, M. (2007). Optimal foot shape for a passive dynamic biped. Journal of Theoretical Biology, 248, 331-339.

Manchester, I. R., Mettin, U., Iida, F., \& Tedrake, R. (2009, in press). Stable dynamic walking over rough terrain: theory and experiment. In Proceedings of the international symposium on robotics research (ISRR2009).

Manoonpong, P., Geng, T., Kulvicius, T., Porr, B., \& Wörgötter, F. (2007). Adaptive, fast walking in a biped robot under neuronal control and learning. PLoS Computational Biology, 3(7), 13051320.

McGeer, T. (1988). Stability and control of two-dimensional bipedal walking. Simon Fraser University CSS-ISS TR 88-01.

McGeer, T. (1990). Passive dynamic walking. International Journal of Robotics Research, 9(2), 62-82.

Miyakoshi, S., \& Cheng, G. (2004). Examining human walking characteristics with a telescopic compass-like biped walker model. In Proceedings of the IEEE international conference on systems, man and cybernetics (SMC2004) (pp. 1538-1543).

Ono, K., Furuichi, T., \& Takahashi, R. (2004). Self-excited walking of a biped mechanism with feet. International Journal of Robotics Research, 23(1), 55-68.

Pekarek, D., Ames, A. D., \& Marsden, J. E. (2007). Discrete mechanics and optimal control applied to the compass gait biped. In Proceedings of IEEE conference on decision and control (pp. 53765382).

Pratt, J., Chew, C.-M., Torres, A., Dilworth, P., \& Pratt, G. (2001). Virtual model control: an intuitive approach for bipedal locomotion. International Journal of Robotics Research, 20(2), 129-143.

Spong, M. W. (2003). Passivity based control of the compass gait biped. In IFAC world congress (pp. 19-24).

Spong, M. W., \& Bhatia, G. (2003). Further results on control of the compass gait biped. In Proceedings of the IEEE international conference on intelligent robots and systems (IROS) (pp. 19331938).

Su, J. L.-S., \& Dingwell, J. B. (2007). Dynamic stability of passive dynamic walking on an irregular surface. ASME Journal of Biomechanical Engineering, 129(6), 802-810.

Taga, G., Yamaguchi, Y., \& Shimizu, H. (1991). Self-organized control of bipedal locomotion by neural oscillators in unpredictable environment. Biological Cybernetics, 65, 147-159.

Tedrake, R. (2004). Applied optimal control for dynamically stable legged locomotion. $\mathrm{PhD}$ thesis, Massachusetts Institute of Technology.

Tedrake, R., Zhang, T. W., \& Seung, H. S. (2004). Stochastic policy gradient reinforcement learning on a simple 3D biped. In Proceedings of the IEEE international conference on intelligent robots and systems (IROS) (Vol. 3, pp. 2849-2854). 
van der Linde, R. Q. (1999). Passive bipedal walking with phasic muscle contraction. Biological Cybernetics, 81, 227-237.

Wisse, M., \& van Frankenhuyzen, J. (2003). Design and construction of MIKE: a 2D autonomous biped based on passive dynamic walking. In Proceedings of international symposium of adaptive motion and animals and machines (AMAM03).

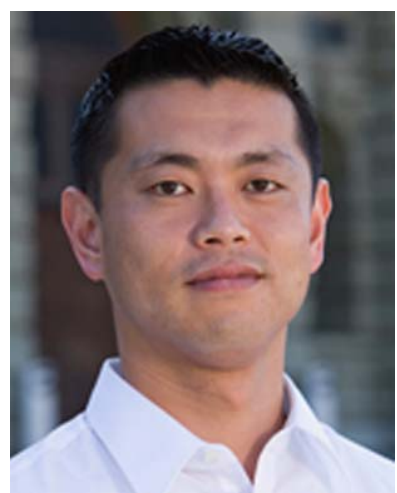

Fumiya Iida received his bachelor and master degrees in mechanical engineering at Tokyo University of Science (Japan, 1999), and Dr.SC. Nat. in Informatics at University of Zurich (2006). In 2004 and 2005, he was also engaged in biomechanics research of human locomotion at Locomotion Laboratory, University of Jena (Germany). From 2006 to 2009 , he worked as a postdoctoral associate at the Computer Science and Artificial Intelligence Laboratory, Massachusetts Institute of Technology in USA. Since 2009, he is an assistant professor at Department of Mechanical and Process Engineering, the Swiss Federal Institute of Technology Zurich (ETH Zurich). In 2006, he awarded the Fellowship for Prospective Researchers from the Swiss National Science Foundation, and in 2009, the Swiss National Science Foundation Professorship. He has been involved in a number of research projects related to robotics, embodied artificial intelligence and biomechanics, and his research interest includes biologically inspired robotics, navigation of autonomous robots, and dynamic legged locomotion. He serves as the editorial board of a few journals and the program committee member of a dozen of international conferences, and he was a recipient of the Prospective Researcher Fellowship of the Swiss National Science Foundation.

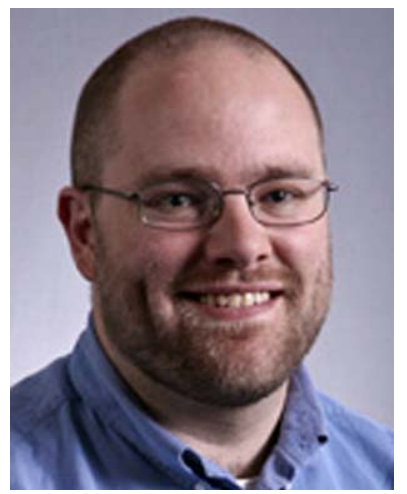

Russ Tedrake is an associate professor of Electrical Engineering and Computer Science at Massachusetts Institute of Technology (USA), and also a member of the Computer Science and Artificial Intelligence Lab. He received an NSF CAREER award, the MIT Jerome Saltzer award for undergraduate teaching, the DARPA Young Faculty Award, and was named a Microsoft Research New Faculty Fellow. He received his B.S.E. in Computer Engineering from the University of Michigan, Ann Arbor, in 1999, and his Ph.D. in Electrical Engineering and Computer Science from MIT in 2004, working with Sebastian Seung. After graduation, he joined the MIT Brain and Cognitive Sciences Department as a Postdoctoral Associate. During his education, he has also spent time at Microsoft, Microsoft Research, and the Santa Fe Institute. The goal of his research is to build machines which exploit their natural dynamics to achieve extraordinary agility and efficiency. This challenge involves a tight coupling between mechanical design and underactuated nonlinear control, and that tools from machine learning and optimal control can be used to produce this coupling when classical control techniques fail. His projects include minimally-actuated dynamic walking on moderate terrain, quadrupedal locomotion on extreme terrain, fixed-wing acrobatics, flapping-winged flight, and feedback control for fluid dynamics. 